\title{
¿POR QUÉ UN DOSSIER SOBRE DESIGUALDAD, DISTRIBUCIÓN Y NIVEL DE VIDA EN EL SIGLO XIX?
}

\section{Daniel Santilli*}

Es indudable que el interés por la medición de la desigualdad y el nivel de vida que ha alcanzado la humanidad ha conseguido una actualidad que hace tiempo no se veía en las ciencias sociales con una temática específica. La preocupación principal son las diferencias que se han generado tanto a nivel individual como regional o entre países. Prácticamente no hay día en que no descubramos en los diarios menciones acerca de este tema, desde diversos ángulos, tanto cultural como económico o social. Diagnósticos académicos sobre la desigualdad en el nivel de vida aparecen casi cotidianamente. ${ }^{1}$ En el mundo académico podemos citar congresos, conferencias, jornadas, seminarios, etc., que dedican sesiones y hasta invocan en su denominación el tema de la desigualdad y el nivel de vida, tanto desde la sociología como desde la economía. Y ha pasado a ser la preocupación, en buena hora, de los gobernantes de la mayor parte del mundo, aunque a veces sea sólo declamada...

Estas disciplinas interrogan a la historia sobre este particular. Sociología, antropología y economía, interpelan a nuestra ciencia acerca del devenir de la desigualdad a través de los tiempos. Y es evidente que la disciplina tiene mucho que decir al respecto. Pero no actúa sólo como reacción a los pedidos de las ciencias sociales, sino de motu proprio, acuciada por los intereses de la historia como ciencia. Como los padres fundadores de la actual historiografía, los historiadores repetimos que no hacemos historia sino actualidad, nuestros intereses tienen más que ver con la realidad cotidiana que con el pasado; ya no somos desenterradores del pasado, anticuarios al decir de Jim Sharpe (1996), sino actores de nuestro tiempo que buceamos en el pasado a partir de preguntas que nos hacemos sobre el presente, desde el presente y para aportar explicaciones al presente.

Es por ello que han florecido los estudios acera de la distribución aplicados a la historia en los últimos años. Tal vez el origen se remonta a la archiconocida cuestión

\footnotetext{
1 Un boom no solo editorial ha sido a ese respecto la publicación del libro de Thomas Piketty (2014) y la secuela de discusiones que ha generado (Acemoglu y Robinson, 2014; Mccloskey, 2014; Obregon, 2015; Zamparelli, 2015). Pero el mérito de Piketty es haber reavivado un avispero que ya existía. Ver por ejemplo (Atikson, 1975; Brenner, Kaelble y Thomas, 1991; Milanovic, 2011) y los monumentales dos tomos del Handbook of income distribution (Atkinson y Bourguignon, 2000).
}

\footnotetext{
Doctor en Historia. Instituto Ravignani-UBA/CONICET. dvsantilli@gmail.com
} 
Santilli. ¿Por qué un dossier sobre desigualdad, distribución y nivel de vida en el siglo XIX?

del nivel de vida británico ${ }^{2}$, que sin embargo siempre es retomada (Allen, 2009; Kelly, Gráda y Mokyr, 2013). O la famosa medición de Kuznets, luego impuesta como ley por los interesados en la teoría de la copa y del derrame (o goteo según la traducción de José Nun, 2011). ${ }^{3}$ Tampoco otros tópicos ya casi clásicos como el nivel de vida en las colonias norteamericanas han caído en el olvido; han sido retomados (Lindert y Williamson, 2016), tal vez motivado por el ya citado trabajo de Piketty.

Pero últimamente se ha incorporado el concepto de multidimensionalidad de los estudios de nivel de vida, derivado directo de la desigualdad, que se agrega a la multidisciplinariedad de dichos estudios. Es decir, no sólo es un tema del que se ocupan, historiadores, economistas, sociólogos y demógrafos, entre otros cientistas sociales, sino que además empiezan a tenerse en cuenta otros aspectos de la desigualdad que influye en el nivel de vida, como la educación, las diferencias de género y las generacionales, por ejemplo (Kessler, 2014), derivados de los estudios de Amartya Sen de veinte años a esta parte. ${ }^{4}$

Pero la historiografía argentina y la latinoamericana, en general han dejado de lado, por ahora, la cuestión de la multidimensión del concepto. Es que aún no se han reconstruido las variables más significativas de las diferencias de ingreso, lo que razonablemente dificulta avanzar en otros aspectos. ${ }^{5}$ Por lo tanto, la mayor preocupación de la literatura al respecto en Argentina es el estudio de la distribución de bienes y de la desigualdad. Los objetivos entonces son, en primer lugar, el análisis del reparto de la riqueza, sobre todo de la tierra, el principal bien de capital durante buena parte del siglo XIX, y en la del ganado, que durante la primera mitad de esa centuria ha sido más importante (Gelman y Santilli, 2006; Gelman, 2011; Santilli, 2016). En segundo lugar, el estudio del ingreso, desde diversos ángulos. En ese sentido los avances más importantes se corresponden al siglo XX, y sobre todo la segunda mitad. Para la etapa 1900-1950 tenemos más estudios de tipo cualitativo, que testimonian las diferencias sociales pero que no construyen validaciones de tipo cuantitativo (Torrado, 2007), con algunas excepciones que recopilan datos y elaboran series sobre variables necesarias para la construcción del nivel de ingreso, como son precios y salarios (Cortés Conde, 1979; Cuesta, 2012; Correa Deza y Nicolini, 2014). Para la segunda mitad, en cambio,

2 Cito los ya trillados trabajos de (Ashton, 2009; Hobsbawm, 2009; Taylor, 1985; Thompson, 1989; Fogel y otros, 1990).

3 Simon Kuznets demostró que la industrialización en Gran Bretaña y Estados Unidos produjo desigualdad en un principio, pero luego el propio desarrollo económico generaba una caída de la desigualdad, en forma autónoma, describiendo una curva en forma de "U" invertida (Kuznets, 1966); esta proposición sirvió para que los defensores de la "ley del derrame" la confirmaran. Se ha demostrado, en primer lugar, que esa ley no es general (Bértola 2005) por lo menos para esta parte del mundo, y, en segundo lugar, la utilidad política que prestó en el transcurso de la guerra fría (Piketty, 2014).

${ }^{4}$ Las Naciones Unidas, a través del PNUD, hace ingentes esfuerzos para incorporar formas de medir esas diferencias. El más antiguo es el Índice de Desarrollo Humano (IDH), pero ha desarrollado el Índice de Pobreza Multidimensional (IPM), el Índice de Desarrollo humano relativo al Género (IDG), el índice de Potenciación de Género (IPG), etc.

5 Una de las excepciones a esta afirmación son los trabajos de Guillermo Velázquez, quien incorpora a sus estudios los aspectos relacionados con la influencia en la calidad de vida con aspectos medioambientales y de vivienda, sanidad, etc. a través de los últimos censos nacionales (Velazquez, 2007). 
los estudios han profundizado en una imagen más global del nivel de ingresos, sobre todo desde una perspectiva diferente, la distribución funcional del ingreso, es decir la distribución por factores, el capital y el trabajo (Lindenboin, Graña y Kennedy, 2005).

Pero el mayor avance se produce con los trabajos sobre las últimas décadas, encarado por economistas y sociólogos, más que por historiadores. En primer lugar, hay que mencionar el aporte metodológico realizado, que puede ser aplicado a la historia previa a la de estas últimas décadas (Gasparini, Cicowiez y Sosa Escudero, 2013; Actis Di Pasquale, 2012; Salvia, 2015). Si bien resulta muy difícil la aplicación de estas herramientas para etapas anteriores a 1900, algunos de sus conceptos pueden ser tenidos en cuenta, con modificaciones metodológicas que lo adecuen a la escasez de fuentes. ${ }^{6}$

En segundo lugar, y más allá de lo metodológico que debe interesarnos para aplicar a períodos anteriores, hay que destacar los trabajos específicos sobre las últimas seis o más décadas (Cruces y Gasparini, 2009a y 2009b; Alvaredo y Piketty, 2011). Pero la mayor atención de los investigadores ha sido la etapa que Kessler (2016) llama postneoliberal, que en Argentina se inicia en el 2002. Infinidad de trabajos han aparecido en estos últimos años, a modo de explicación y otros a modo de balance, que podríamos enmarcar, desde el punto de vista historiográfico, en la historia reciente ${ }^{7}$.

Pero el objetivo de nuestro dossier es el siglo XIX, el largo siglo XIX, que comienza tal vez con la creación del Virreinato del Río de la Plata y concluye en 1914, en plena "canción de otoño en primavera", al decir de Tulio Halperín Donghi. Presentamos aquí seis trabajos que por supuesto no agotan el panorama historiográfico de la historia económica del siglo XIX y ni siquiera la amplitud de los estudios sobre desigualdad y nivel de vida del período. ${ }^{8}$ Pero abarcan diversos aspectos de este proceso y de sus avances parciales, desde el estudio de la distribución de la tierra a la construcción de canastas de consumo, pasando por la elaboración de series de precios y de salarios, así como por diferentes regiones de la futura Argentina.

El primer trabajo que presentamos es el de Julio Djenderedjian y Juan Luis Martirien. Se trata de un estudio de precios a partir de las alcabalas, para fines del siglo XVIII en varias ciudades del entonces Virreinato. No es común indagar sobre precios a partir de este impuesto que grava todas las operaciones, no importa en qué grado de la cadena se produce. Es decir, el impuesto es pagado tanto por el vendedor-productor,

\footnotetext{
${ }_{6}$ Por ejemplo, la distribución funcional del ingreso podría ser usada aplicando las formulaciones del teorema de Heckscher-Ohlin y de la derivación de Williamson (O' Rourke, Taylor y Williamson, 1996), del cual se deduce que el aumento del precio de la tierra implica un incremento de la retribución al capital invertido en tierras, en detrimento del trabajo (Gelman y Santilli, 2015).

7 A modo de ejemplo y sin intentar agotar las obras, mencionamos trabajos de Cruces y Gasparini y su grupo (2010), de Salvia y sus dirigidos (Salvia, Tuñon y Poy, 2015). Los trabajos del grupo dirigido por Javier Lindenboim (2008) y del coordinado por Demián Panigo (De Giovambattita, Gallo y Panigo, 2014). Últimamente han aparecido trabajos que reflexionan acerca del balance de esos años postneoliberales (Lindenboim y Salvia, 2015; Kulfas, 2016; Kessler, 2016), reflexión que necesariamente se extiende a América Latina en conjunto (López-Calva y Lustig, 2011). Una profunda aseveración sobre la historia económica de América Latina teniendo en cuenta el crecimiento y la distribución puede verse en (Bértola y Ocampo, 2013).

8 Un resumen de los avances y de los temas pendientes al respecto, en Guzman y Santilli, 2013.
} 
como por el intermediario, como por el que pasa por la ciudad, como por el que la introduce para su venta por menor. De todos modos, como dicen sus autores sirve como punto de referencia, para marcar tendencias y diferencias entre localidad y localidad. Lo notable del trabajo es la relativa convergencia de las tendencias de las variaciones de precios, que indicaría cierto correlato en la conformación de precios entre las regiones. Queda pendiente la comparación de los precios nominales, por cierto. Si bien estos son precios mayoristas, la posibilidad de establecer precios al consumidor está más cerca; y sobre todo se aplica un innovador modo de acercarse a los mismos hasta ahora muy poco utilizado.

La segunda intervención corresponde a la ciudad de Tucumán entre 1816 y 1850 , y se debe a la autoría de María Paula Parolo. A través del estudio de precios obtenidos en diversas fuentes de índole pública y privada, se construyen índices de precios minoristas para productos de consumo masivo, como maíz, arroz, azúcar, carbón y leña, etc. Para el ganado, en cambio, las observaciones son en su fase mayorista. Lo que se destaca es la intervención del Estado en la fijación de los precios desde su papel asignado en el Antiguo Régimen, para proveer tranquilidad social, combatiendo la especulación a la que son proclives algunos comerciantes. De modo que los modos de regulación no son los que puede proveer el librecambio, derechos aduaneros, impuestos diferenciados, etc. Asimismo, el trabajo demuestra las variaciones originadas en condicionantes provocados por las guerras, tanto desde el punto de vista de la demanda como de la oferta. También contribuyeron a esas variaciones condiciones climáticas. El trabajo entonces es un paso previo a la construcción de índices de precios combinados, material necesario para estudiar el consumo de los sectores populares, o de la población en su conjunto, y proveer a la indagación acerca del nivel de vida de los pobladores.

El tercero es nuestro trabajo, en conjunto con Jorge Gelman, acerca de la construcción de canastas para la ciudad de Buenos Aires para la primera mitad del siglo XIX. Se trata de un análisis de la metodología ideada por Robert C. Allen para comparar diversas geografías a través del tiempo, teniendo en cuenta un consumo básico de calorías considerado igual a través de esas variables. El trabajo analiza las particularidades y dificultades de la aplicación del modelo a nuestra realidad e incluye un análisis puntual comparativo con el estudio efectuado sobre la misma ciudad en el mismo tiempo por otros investigadores, con resultados muy diferentes a los obtenidos por nosotros, explicado precisamente por las diferencias generadas en la aplicación de la metodología en cuestión. En definitiva, un llamado de atención con respecto a la aplicación automática de metodologías ideadas para otros espacios y momentos, y con un objetivo que puede ser diferente al buscado por nosotros. Queda pendiente una constatación de la perdurabilidad de la misma canasta a través del período en estudio, es decir el largo siglo XIX

Le sigue el artículo escrito por Roberto Schmit, quien estudia los precios de los bienes importados en Buenos Aires, entre 1824 y 1850 . El autor expone las oscilaciones que sufren estos precios medidos en la moneda corriente de Buenos Aires, el peso papel, provocados por los procesos inflacionarios desatados a partir de 1825, 
cuyo desencadenante fue el bloqueo del puerto en ese año y en 1838/40. Este resultado es mucho más notorio y más volátil después del segundo bloqueo y se extendió a toda la década de 1840. Sin embargo, cuando se los analiza en moneda constante, las variaciones no son tan pronunciadas y la tendencia es mayormente a la baja. En este sentido, debe anotarse que una de las razones puede ser la tendencia a la baja de los precios en origen por la creciente industrialización, o por lo menos la reducción en el precio del transporte, como en el caso del azúcar. El otro motivo para la tendencia a la baja puede tener con la elasticidad de la demanda que, ante un aumento de precios y la consiguiente pérdida de poder adquisitivo, ya que los sueldos no acompañaban, retrae el consumo. Un trabajo necesario para la construcción de la canasta y el estudio del nivel de vida, medida a través del ingreso.

El siguiente es el estudio de Beatriz Bragoni y Patricia Olguín sobre los salarios en momentos del gran auge vitivinícola en Mendoza. Las autoras discuten con la historiografía que analiza los salarios reales en esa ciudad entre fines del siglo XIX y principios del XX. En el trabajo se demuestra que para los empleados del Estado éstos se mantuvieron, pero no crecieron. Sin embargo, no pasó lo mismo con los relacionados con la producción vitivinícola, el sector más dinámico de la economía. En efecto, los salarios de los peones de la empresa vitivinícola analizados cayeron en términos reales por lo menos en la segunda mitad del período en análisis, contradiciendo las versiones previas. Asimismo, muestran que todos los salarios habrían caído en comparación con la recaudación fiscal, por lo que, si se toma esta última como un proxy del PBI, la distribución funcional del ingreso también se habría deteriorado. Sin embargo, el Estado pagaba mejores sueldos que los sectores más dinámicos de la economía mendocina. ¿Es que este actor asume que a través de esa función contiene las desigualdades sociales? ¿O de algún modo se adjudica funciones redistributivas que no realiza el mercado pero que además no se las pide? No lo sabemos aún. Bragoni y Olguín concluyen acerca de la necesidad de profundizar los estudios.

Por último, presentamos el trabajo de Cecilia Fandos sobre los arrendatarios de Cochinoca en Jujuy, a fines del siglo XIX. La autora discute también con cierta bibliografía que estimaba que los arrendatarios eran sujetos pasivos de una explotación desmesurada y ancestral, y que los condenaba a la indefectible proletarización una vez finiquitada la hacienda del Marqués de Tojo, propiedad excluyente de la región, con la expropiación por parte del Estado. Las condiciones del arrendamiento se suavizaron y los pobladores pudieron mínimamente mejorar su nivel de ingreso, al punto de lograr un incremento demográfico y una mínima acumulación, a la luz del aumento del stock ganadero en manos de los arrendatarios. Cuando nuevamente se privatizó la tierra, las condiciones empeoraron en cuanto a la distribución de la tierra, precisamente. Sin embargo, aunque aún no hay evaluaciones cuantitativas sobre el nivel de ingresos de esta etapa, los testimonios cualitativos indican que empeoró, ya que los pobladores añoraban la época del arriendo fiscal, es decir cuando la tierra estaba en manos del Estado. El artículo es, por tanto, un paso necesario en el esclarecimiento de las condiciones de vida en la región. 
Es imposible no hacer paralelos y reflexionar acerca de la acción del Estado en la tarea de matizar las desigualdades, tarea llevada a cabo en plena etapa liberal, en pleno apogeo de las ideas liberales de obligatoria prescindencia del mismo. Como vemos, taxativa o sin que los dirigentes lo propongan explícitamente, el Estado trata de limar las aristas más agudas de la desigualdad, sin dejar de favorecer en muchos casos a los sectores más dinámicos de la economía.

Todavía no podemos asegurar que el nivel de vida mejoró en estos casos estudiados, ya que un supuesto incremento en el ingreso no se traduce definitivamente en una mejora del nivel de vida, aun no teniendo en cuenta lo relativo del concepto. Sin embargo, a la luz de una de las pocas metodologías que se han propuesto medir el nivel de vida de determinada población en un momento dado, la antropometría, se comprueba que, por lo menos en Buenos Aires, los niños nacidos y/o criados en la provincia en la primera mitad del siglo, aumentaron de estatura, signo inequívoco de dicha mejora (Salvatore, 2004; 2007). Ello más allá de todas las dudas que genera la metodología de las estaturas.

La agenda pendiente, como se mencionó con anterioridad y como se desprende de varios de los textos presentados, se construye con la necesidad de más trabajos que elaboren series de precios y sueldos y más investigaciones acerca de la canasta de cada espacio de la futura Argentina. Y además alguna aproximación al ingreso de los campesinos, es decir su producción y la rentabilidad de esas pequeñas explotaciones, de las que vivía la gran mayoría de la población, inmensamente rural aún. Por último, mas búsqueda de testimonios cualitativos acerca de cómo vivían, que comían, costumbres, etc. de los sectores populares, a fin de evitar caer en anacronismos.

\section{Referencias bibliográficas}

Acemoglu, D. y Robinson, J. 2014. The rise and decline of general laws of capitalism. NBER Working Paper Series, Cambridge: National bureau of economic research.

Actis Di, P. E. 2012. Bienestar social, resignificación del concepto y de su operacionalización. Un aporte metodológico apliacado al caso argentino. Tesis de doctorado, Bernal: UNQ.

Allen, R. C. 2009. The British industrial revolution in global perspective. Cambridge: Cambridge University Press, Ebook edition.

Alvaredo, F. y Piketty, T. 2011. «La dinámica de la concentración del ingreso en países desarrollados y en desarrollo. Una perspectiva desde los altos ingresos.» En: López, L. F.; Calva Lustig, N. (comps.) La disminución de la desigualdad en América Latina. ¿Un decenio de progreso?, México: FCE, 109-146.

Ashton, T. S. 2009. "El nivel de vida de los trabajadores en Inglaterra. 1790-1830". En: Quiroz, E. (comp.) Consumo e historia. Una antología, México: Instituto Mora, 27-53.

Atkinson, A. B. 1975. The economics of inequality. Oxford: Clarendon Press.

Atkinson, A. B.; Bourguignon, F. (edits.). 2000. Handbook of income distribution. Amsterdam: Elsevier. 
Bértola, L. 2005. "A 50 años de la curva de Kuznets. Crecimiento económico y distribución del ingreso en Uruguay y otras economías de nuevo asentamiento desde 1870". En: Investigaciones de Historia Económica, № 3, 135-176.

Bértola, L. y Ocampo, J. A. 2013. El desarrollo económico de América Latina desde la independencia. México: FCE.

Brenner, Y. S, Hartmut Kaelble, y Mark Thomas (edits.). 1991. Income distribution in historical perspective. Cambridge: Cambridge University Press.

Correa Deza, M. F. y Nicolini, E. A. 2014. "Diferencias regionales en el costo de vida en Argentina a comienzos del siglo XX”. En: Investigaciones de Historia Económica, No 10, 202-212.

Cortés Conde, R. 1979. El Progreso Argentino, 1880-1914. Buenos Aires: Sudamericana.

Cruces, G. y Gasparini, L. 2009a. "Desigualdad en Argentina. Una revisión de la evidencia empírica: Primera Parte”. En: Desarrollo Económico 48, N 192, 395437.

Cruces, G. y Gasparini, L. 2009b. "Desigualdad en Argentina. Una revisión de la evidencia empírica: Segunda Parte". En: Desarrollo Económico 49, No 193, 3-29.

Cuesta, E. M. "Precios y salarios en Buenos Aires durante la gran expansión (18501914)". En: Revista de Instituciones, Ideas y Mercados, № 56, 159-179.

De Giovambattista, A.; Gallo, P. y Panigo, D. 2014. "El impacto distributivo del "PROG.R.ES.AR" en Argentina. Una primera aproximación en base a microsimulaciones". En: Empleo, desempleo \& politicas de empleo, No 17, 191.

Fogel, R. y otros. 1990. "Cambios seculares en la estatura y la nutrición en Estados Unidos y Gran Bretaña”. En: Rotberg, R. I. y Rabb, T. K. (comps.) El hambre en la historia, Madrid: Siglo XXI, 271-312.

Gasparini, L. y Cruces, G. 2010. "Las asignaciones universales por hijo en Argentina. Impacto, discusión y alternativas”. En: Económica, LVI, 105-146.

Gasparini, L.; Cicowiez, M. y Sosa Escudero, W. 2013. Pobreza y desigualdad en América Latina. Conceptos, herramientas y aplicaciones. Buenos Aires: Temas/ CEDLAS.

Gelman, J. (Comp). 2011. El mapa de la desigualdad en la Argentina del siglo XIX. Rosario: Prohistoria.

Gelman, J. y Santilli, D. 2006. De Rivadavia a Rosas. Desigualdad y crecimiento económico. Buenos Aires: Siglo XXI.

Gelman, J. y Santilli, D. 2015. "Salarios y precios de los factores en Buenos Aires, 17701880. Una aproximación a la distribución funcional del ingreso". En: Revista de Historia Económica - Journal of Iberian and Latin American Economic History, No $33,153-186$.

Guzman, T. y Santilli, D. 2013. "Las condiciones de vida de los pobres en la futura Argentina. El largo siglo XIX”. En: Di Meglio, G. y Fradkin , R. (comps.) Hacer política. La participación popular en el siglo XIX rioplatense, Buenos Aires: Prometeo, 363-396.

Hobsbawm, E. J. 2009. “El nivel de vida británico, 1790-1850”. En: Quiroz, E. (Comp.) Consumo e histgoria. Una antología. México: Instituto Mora, 287-328. 
Kelly, M.; Gráda, C. y Mokyr, J. 2013. Precious Albion: a new interpretation of the British industrial revolution. Working Paper Series, Dublin: UCD CENTRE FOR ECONOMIC RESEARCH.

Kessler, G. (Comp.) 2016. La sociedad argentina hoy. Radiografia de una nueva estructura. Buenos Aires: Siglo XXI.

Kessler, G. 2014. Controversias sobre la desigualdad. Argentina 2003-2013. Buenos Aires: FCE.

Kulfas, M. 2016. Los tres kirschnerismos. Una historia de la economía argentina. 20032015. Buenos Aires: Siglo XXI.

Kuznets, S. 1966. Modern Economic Growth. New Haven: Yale University Press.

Lindenboim, J. y Salvia, A. (Comp). 2015. Hora de balance: Proceso de acumulacion, mercado de trabajo y bienestar. Bienos Aires: Eudeba.

Lindenboim (comp.), Javier. Trabajo, ingresos y políticas en Argentina. Contribuciones para pensar el siglo XXI. Buenos Aires: Eudeba, 2008.

Lindenboin, J.; Graña, J. M. y Kennedy, D. 2005. Distribución funcional del ingreso en la Argentina. Ayer y hoy. Buenos Aires: CEPED. Documento de Trabajo No 4.

Lindert, P. H., y Williamson, J. 2016. American Growth and Inequality since 1700. Unequal Gains. Princeton and Oxford: Princeton University Press.

López-Calva, L. F. y Lustig, N. (comps.) 2011. La disminución de la desigualdad en América Latina. ¿Un decenio de progreso? México: FCE.

McCloskey, D. N. 2014. "Measured, Unmeasured, Mismeasured, and Unjustified Pessimism: A Review Essay of Thomas Piketty's Capital in the Twenty-First Century". Erasmus Journal for Philosophy and Economics 7, № 2, 73-115.

Milanovic, B. 2011. Los que tienen y los que no tienen. Una breve y singular historia de la desigualdad global. Madrid: Alianza.

Nun, J. 2011. "La riqueza no gotea ni derrama". La Nación, 22 de Junio.

Obregon, C. 2015. Piketty is wrong. Paper, MPRA.

O'Rourke, K. H., Taylor, A. M. y Williamson, J. G. 1996. "Factor price convergence in the late nineteenth century". International Economic Review, $\mathrm{N}^{\mathrm{o}} 37,499-530$.

Piketty, T. 2014. El capital en el siglo XXI. México: FCE.

Salvatore, R. 2004. "Alturas, nutrición y bienestar en la Argentina (1790-1950). Algunos hallazgos recientes y su importancia para la historia económica". XIX Jornadas de Historia Económica. San Martín de los Andes.

Salvatore, R. 2007. "Heights, nutrition and well-being in Argentina, ca. 1850-1950. Preliminary results". En: Revista de Historia económica, XXV, $\mathrm{N}^{\mathrm{o}}$ 1, 53-86.

Salvia, A. 2005. El desarrollo humano y la segmentación social de los espacios urbanos. El marco teórico y metodológico de una aplicación útil al diseño de políticas. Documentos de Monitoreo de la deusa social argentina, Buenos Aires: Universidad Católica Argentina.

Salvia, A.; I. Tuñon, y S. Poy. 2015. "Asignación Universal por Hijo para Protección Social: impacto sobre el bienestar económico y el desarrollo humano de la infancia”. En: Población y Sociedad 22, N² 2, 101-134. 
Santilli, Daniel. «El precio de la "modernidad": La evolución de la desigualdad en la propiedad de la tierra en la campaña de Buenos Aires, 1839-1914.» Historia Agraria, $\mathrm{n}^{\circ} 69$ (2016): 73-103.

Sharpe, J. 1996. "Historia desde abajo". En: Burke, P. Formas de hacer historia, Madrid: Alianza, 38-58.

Taylor, A. 1985. El nivel de vida en Gran Bretaña durante la revolución industrial. Madrid: Ministerio del Trabajo y Seguridad Social.

Thompson, E. P. 1989. La formacion de la clase obrera en Inglaterra. Barcelona: Crítica.

Torrado, S. (comp.). 2007. Población y bienestar en la Argentina del primer al segundo centenario. Buenos Aires: Edhasa.

Velazquez, G. 2007. Población, territorio y calidad de vida. Vol. II, de Población y bienestar en la Argentina del primero al segundo centenario. Una historia social del siglo XX. En: Torrado S. (comp.), Buenos Aires: Edhasa, 573-600.

Zamparelli, L. 2015. Wealth Distribution, Elasticity of Substitution, and Piketty: an antidual Pasinetti Economy. Workin Paper, Roma: Sapienza Università di Roma. 